\title{
INDUCED RADIOACTIVITY AND ITS RELATION TO BEAM LOSSES \\ IN THE CERN 26 GEV PROTON SYNCHROTRON
}

\author{
A.H. Sullivan \\ CERN, 1211 Geneva, 23 \\ Switzerland
}

\begin{abstract}
The results of induced radioactivity measurements made over the past 10 years around the CERN $26 \mathrm{GeV}$ proton synchrotron are presented. The dose rate near different sections of the machine is shown to vary by factors up to 10 above and below the mean. A correlation is made between beam losses and radiation level, where it is estimated that to a first approximation in a machine that has been running a few years, the dose rate at $50 \mathrm{~cm}$ from a straight section between magnets and 24 $\mathrm{h}$ after stop will be $1.0 \mathrm{mSv} / \mathrm{h}(100 \mathrm{mrem} / \mathrm{h})$ for beam losses equivalent to $1 \mathrm{w}$ per metre of machine circumference. The dose rate after a cooling time of $t$ days ( $t<1$ year) is derived to be:

$$
D=p\left(1-0.4 \log _{10} t\right) \mathrm{mSv} / \mathrm{h}
$$

where $p$ is the average beam power loss, in $w$ per $m$ of circumference, over the two preceding months. This dependence of dose rate on decay time is compared with measured data from the PS for up to 43 days of cooling time. Beam losses estimated from induced activity dose rates using the above relation are shown to correspond reasonably with those expected for two operating conditions of the CERN $26 \mathrm{GeV}$ proton synchrotron.
\end{abstract}




\section{INTRODUCTION}

The CERN $26 \mathrm{GeV}$ proton synchrotron (PS) has been in operation for over 25 years, during which time it has been subject to many improvements resulting in higher beam intensities and reductions in maintenance time. A major consideration for the maintenance of the machine is the local radiation level due to induced radioactivity. This activity depends on many factors, not all of which are under control, making its prediction very uncertain. Experience of the systematics of the radioactivity has been acquired from routine measurements around the machine over many years. Given the possibilities of building higher intensity synchrotrons and the present trend towards lowering dose limits for maintenance personnel, an analysis of the available data, even if very approximate, could prove worthwhile for assessing the limits that could be imposed on machine operation by induced radioactivity problems.

\section{INDUCED RADIOACTIVITY LEVELS IN THE PS}

The radiation levels near to the machine are measured at the start of each shut-down, primarily in order to be able to give appropriate advice concerning precautions that may be necessary. The dose rate measurements are made at $40 \mathrm{~cm}$ from the vacuum chamber (approximately $50 \mathrm{~cm}$ from the beam line) near the entrance and exit of each of the 100 bending magnets around the ring. For reasons of convenience measurements are normally made 32 hours after the machine has stopped working. The 200 measurements thus made give a detailed picture of how the radiation levels vary with position and comparison of consecutive surveys show where local loss points are occurring. The average of these measurements, which gives the average dose rate near the straight sections between magnets, is a measure of the overall activation by beam losses in the machine. The evolution of this value over the last 10 years is plotted in Fig. 1, where it can be seen that levels have decreased by more than a factor of 2 in spite of increases in machine utilisation (1). This improvement in efficiency is due to the abandon of internal targets, the installation and continuous improvement of a computer control system and an effort to limit the number of protons that are lost during machine studies.

Over the last few years the PS has been mainly devoted to supplying protons to the super-Proton synchrotron (SPS) by a continuous transfer process (CT) currently at $14 \mathrm{GeV}$ and to supplying protons at $26 \mathrm{GeV}$ for the antiproton production facility, where a fast extraction (FE) system is necessary. A small fraction (less than $1 \%$ ) of protons 
have also been supplied for low intensity test beams by a slow extraction process. The distribution of protons between the principal modes of operation, expressed as maximum beam current available and the average over the years 1985 and 1986, is given in Table 1. The variation of induced activity dose rate over the same period with an indication of the principal mode of PS operation preceeding a shut-down is shown in Fig. 2. There does not appear to be any strong correlation between activity level and mode of operation, although there could be a tendency for the activity to rise during CT operation and to fall after the fast ejected beam has been used. The distribution of activity around the machine after periods of CT and FE operation is shown in Fig. 3, where the position of important loss points can be seen. The dose rate near individual straight sections varies by a factor of up to 10 above and below the mean.

Table 1

Beam intensities from the PS for the principal modes of operation during 1985 and 86

\begin{tabular}{|c|c|c|c|c|}
\hline \multirow{3}{*}{ Mode } & \multirow{3}{*}{$\begin{array}{c}\text { Energy } \\
\text { GeV }\end{array}$} & \multicolumn{3}{|c|}{ Beam intensity (nA) } \\
\hline & & Max. & Ave & rage \\
\hline & & & 85 & 86 \\
\hline Continuous Transfer (CT) & 14 & 330 & 90 & 130 \\
\hline Fast Ejection (FE) & 26 & 1100 & 350 & 240 \\
\hline
\end{tabular}

\section{DEPENDENCE OF THE DOSE RATE ON IRRADIATION CONDITIONS}

The level of induced radioactivity depends on the activating high-energy particle flux $\phi$, the time of irradiation $T$, and the time, $t$, that has elapsed since the irradiation stopped. It has been shown that, to a first approximation, these quantities can be connected by a relation (2):

$$
A=k \phi \log \left(\frac{T+t}{t}\right)
$$

where $T$ and $t$ are in common units and are in the range between about 1 minute and 2 years. $\mathrm{k}$ is a normalizing constant which for precisely known irradiation conditions can be calculated (3). 
In a high-energy particle accelerator the activity will be better related to the radiation energy dissipated than to the activating flux, which will be given by the product of the number of protons lost and the particle energy. The above expression will only be strictly valid when the activating flux is held constant over a well defined period, which is rarely the case in an accelerator where the time and position of beam losses is a matter of conjecture. Even so the relation can be used to make a first order estimate of the dose rate and its variation with time when beam losses are averaged over the period ( 2 months) immediately preceding a shut-down. As can be seen in Table 1, long-term average beam intensities are very much less than the maximum during a run so that it becomes necessary to limit the irradiation time $T$ to prevent undue overestimation of long-term activity. An effective irradiation time for an accelerator that has been in operation for many years will be about 1 year. With these conditions Eq. (1) predicts that the last 2 months operation will contribute $70 \%$ to the activity at 24 hours after stop and about $50 \%$ after 14 days decay. However, as was shown in Fig. 2, the activation is only slightly dependent on mode of PS operation and the approximation for the irradiation conditions should remain reasonably valid for decay times up to at least one month.

The constant to be used to relate dose rate to irradiation conditions can only be approximately calculated, but probably to an accuracy at least as great as that to which the real beam losses in a machine can be estimated. The constant relating activation to particle flux has been determined in Ref. 3. If it is further assumed that:

1) $200 \mathrm{MeV}$ of beam energy are dissipated in a high-energy particle interaction in iron or copper.

2) $10 \%$ of the lost radiation energy is deposited in the machine sections between magnets (the rest being buried in the magnet yokes and shielding).

3) Self shielding of the gamma rays in the straight sections is negligible.

then a calculated dose rate of $1.0 \mathrm{mSv} / \mathrm{h}(100 \mathrm{mrem} / \mathrm{h})$ at $50 \mathrm{~cm}$ and 24 hours after beam off for a beam power loss of $1 \mathrm{~W}$ per metre of circumference is obtained. Such a dose rate constant should be approximately valid for any proton synchrotron. Putting the dose rate constant and irradiation time into Eq. 1, the average dose rate at a time $t$ days after stop when $t$ is very much less than 1 year, approximates to

$$
D=p\left(1-0.4 \log _{10} t\right) \mathrm{mSv} / \mathrm{h}
$$


where $\mathrm{p}$ is the beam power loss in $\mathrm{W} / \mathrm{m}$ in the machine during the period leading up to the shut-down.

This relation is only valid if the activation has been produced at a reasonably constant rate and can be upset if heavy losses have occurred for a short period just before shut-down. In this case the dose rate due to the exceptional losses should be assessed separately and will be given by:

$$
D=\frac{2 J}{t} \mu \mathrm{Sv} / \mathrm{h}
$$

where $\mathrm{J}$ is the beam energy loss in kilojoule per metre of machine, and $t$ is the time since the beam loss in days. (In the PS one $\mathrm{kJ} / \mathrm{m}$ is equivalent to a beam loss of $1.5 \times 10^{14}$ protons at $26 \mathrm{GeV}$ ).

The dependence of dose rate on cooling time has been measured near three PS sections at times up to 43 days after beam off and is compared with Eq. 2 in Fig. 4. As can be seen the $\log t$ dependence is reasonably correct but deviations from the predicted decay occur over limited time periods, indicating the approximate nature of the analysis.

\section{ESTIMATION OF BEAM LOSSES FROM INDUCED ACTIVITY MEASUREMENTS}

The relation between dose rate and beam loss allows crude estimates to be made of the beam losses attributable to the different modes of PS operation. As can be seen in Fig. 2, the average dose rate at 32 hours was $0.32 \mathrm{mSv} / \mathrm{h}(32 \mathrm{mrem} / \mathrm{h})$ at the end of 1985 after a period of fast ejection operation and was also $0.32 \mathrm{mSv} / \mathrm{h}$ at the end of a period devoted to continuous transfer ending in November 1986. These dose rates imply an effective beam power loss of $0.2 \mathrm{~kW}$ in both cases which corresponds to about $1 \%$ of the fast ejected beam and $5 \%$ of the CT beam that was used. The losses estimated in this way are expected to vary from run to run as they include not only those during injection, acceleration and extraction of the beam, but also the protons lost during machine optimization studies as well as various otherwise unrecorded losses due to occasional malfunction of the machine. The magnitude of the losses is within range of those reasonably expected for the two modes of PS operation although in the case of the fast extracted beam the occasional use of the slow extraction 
system appears to make a significant contribution to the apparent beam losses as can be seen in the dose-rate distribution in Fig. 3.

Hence, although the analysis presented is inexact, a first order estimate of average induced activity dose rates from beam losses and vice versa seems possible which, when coupled to the observed factor of 10 variation in dose rate around the machine, should be sufficient to make a reasonable estimate of the conditions under which induced radioactivity will seriously hinder the maintenance of a proton synchrotron.

\section{ACKNOWLEDGEMENTS}

The systematic measurements of induced radioactivity over the past years were made by $E$. Barbe, whose conscientious work has made a quantitative analysis possible. This analysis was stimulated by discussions with $Y$. Baconnier, PS Ring Group, whose interest was appreciated.

\section{REFERENCES}

1. G. Azzoni and K. Priestnall, CERN/PS 86-3 (OP) CERN (1986).

2. A.H. Sullivan and T.R. Overton, Health Physics, 11, 1101 (1965).

3. A.H. Sullivan, Health Physics 23,253 (1972). 


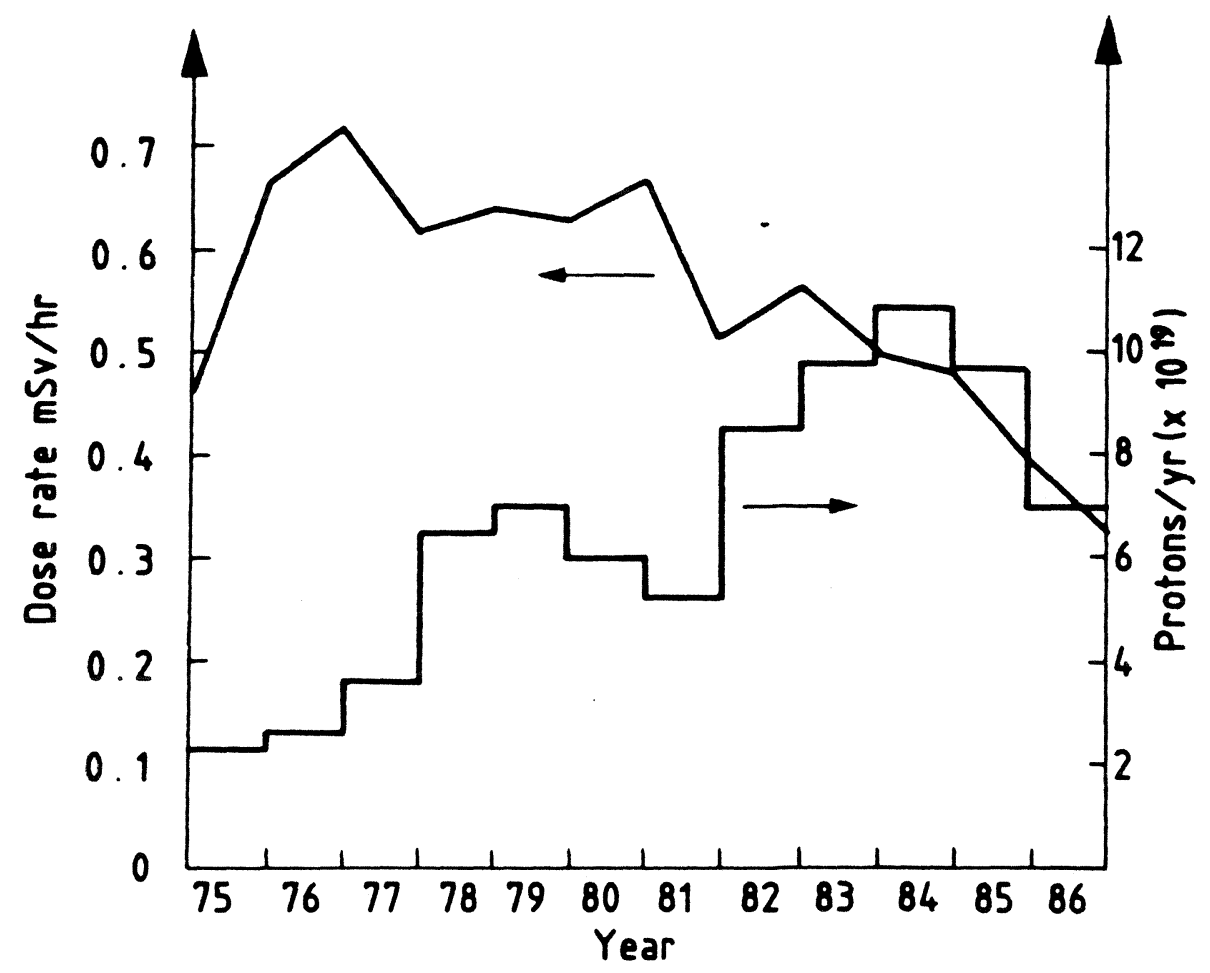

Fig. 1 The evolution of induced activity dose rates since 1975 . Dose rates are the average of those measured at $40 \mathrm{~cm}$ from vacuum chamber and at $32 \mathrm{~h}$ after machine stop. Also shown is the total number of protons accelerated per year for comparison.

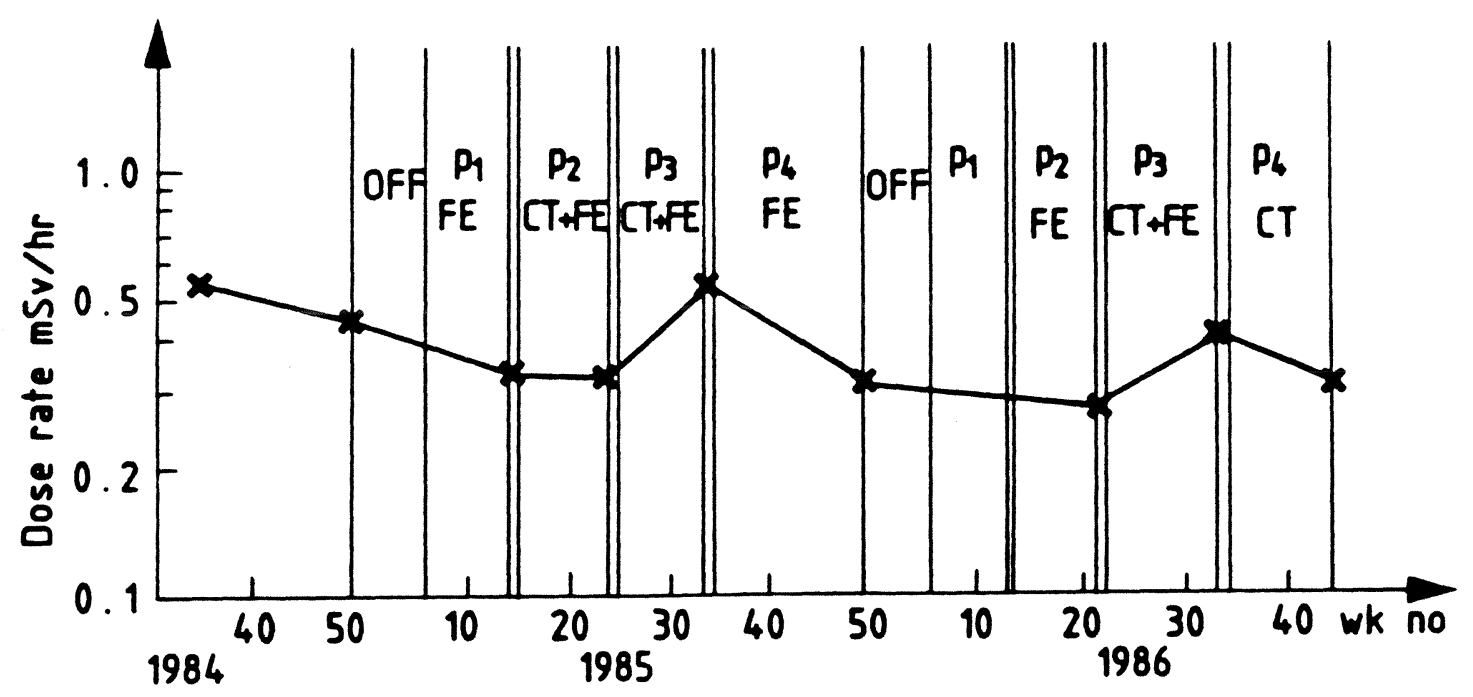

Fig. 2 Induced activity dose rate measured 32 hours after shutdown and its dependence on mode of PS operation. 


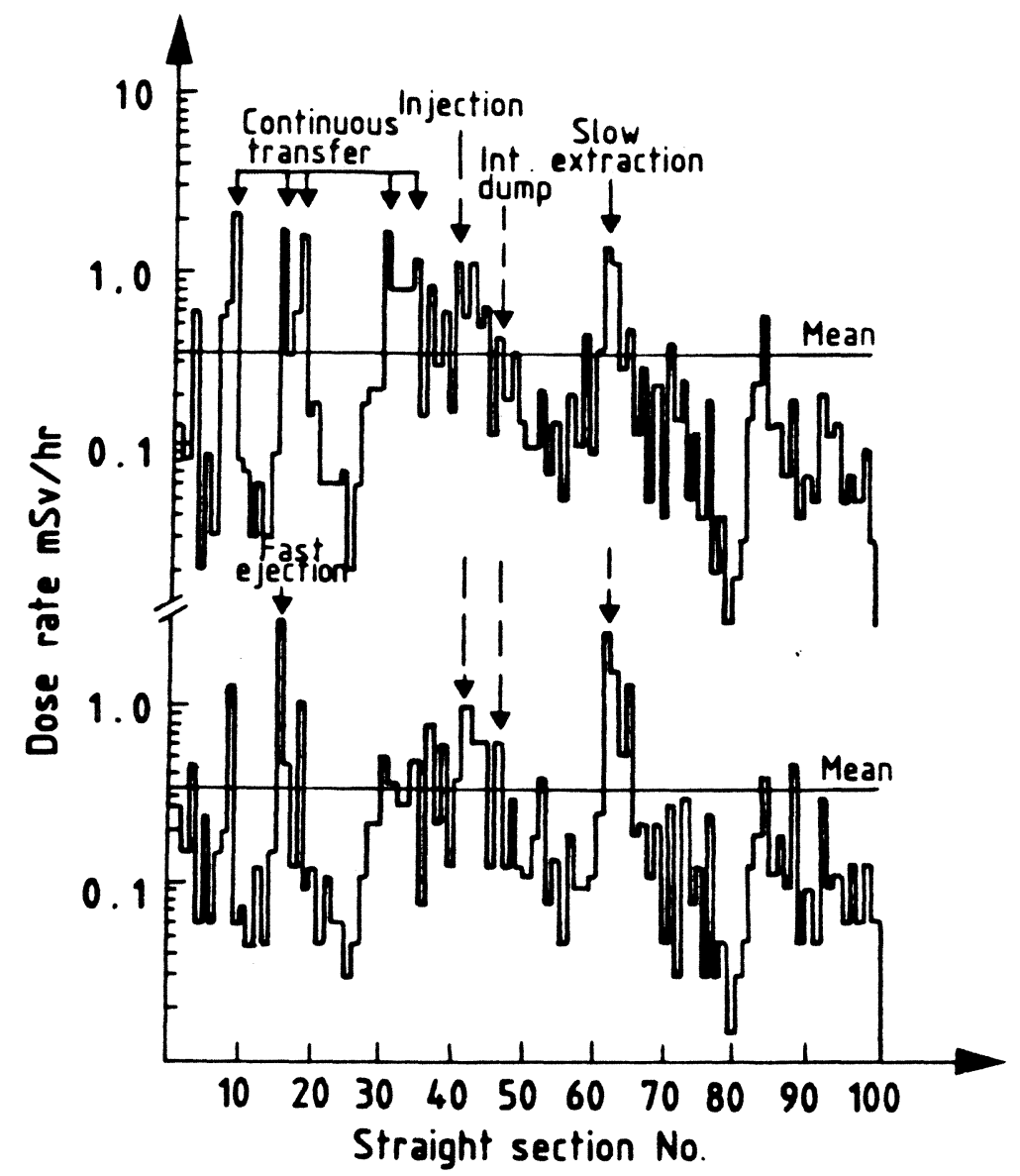

Fig. 3 The distribution of dose rate from induced activity around the PS ring after periods of continuous transfer operation (upper line) and fast ejection. The expected positions of beam losses are indicated for the two modes of operation.

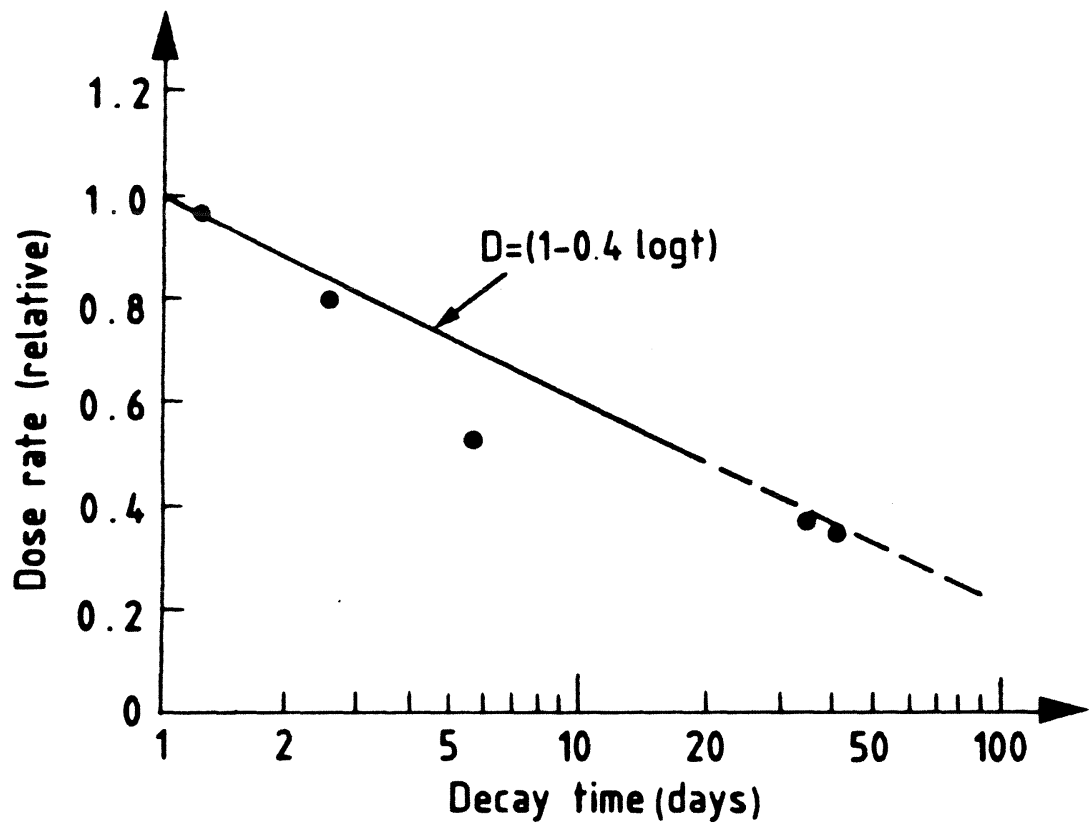

Fig. 4 Dose rate variation with decay time measured over three straight sections of the PS machine compared to the expected decay law. The dose rates have been normalized to unity at 1 day decay time. 